\title{
Using Subspace Pursuit Method For Radar Image Reconstruction
}

\author{
Yueyuan Zhang \\ School of Information Engineering \\ East China Jiaotong University \\ Nanchang, China \\ zyyaney@ecjtu.edu.cn
}

\begin{abstract}
A compressive sensing based method is proposed for reconstruction of radar image of 3-D geometry in this paper. Using the sparse property of the geometry, compressed sensing performs a low-dimensional, nonadaptive and linear projection to acquire an efficient representation of a compressible signal directly using just a few measurements. In order to characterize the problem sparsely, the delta function is used as the sparse basis function. When testing by the incident plane wave at several different frequency points over wide band, linear equations are constructed following the theory of compressed sensing to recover the range profile of radar image. Subspace pursuit (SP) method is applied to solve the linear equations and the results are used for traditional Back-Projection (BP) imaging method. The physical optics is used to compute the scattering which is considered as the input data for compressed sensing based construction. Numerical results of several geometries show our method is robust and accuracy for radar imaging with sub-sampled data.
\end{abstract}

Keywords- Compressive sensing; subspace pursuit method; back projection; radar imaging; sparse reconstruction

\section{INTRODUCTION}

Radar imaging is a kind of inverse scattering problems where by a spatial map of reflectivity is reconstructed from measurements of scattered electric fields. As an active and coherent microwave imaging system with high resolution, radar has the capability to image all weather and day-night conditions. It transmits wide-band signals and receives echoes which are sampled into digital data. The A/D converter could be complicated and expensive since its very high sampling rate. Furthermore, it is challenged for $\mathrm{A} / \mathrm{D}$ in imaging radar to achieve the requirement of sampling wide-band signals $[1,2]$.

In compressive sensing applications, linear program techniques play an important role in designing computationally tractable compressive sensing decoders. However, the complexity of the linear program is still high for many applications. Several classes of low-complexity reconstruction techniques were recently proposed to take the place of linear programming based algorithm, such as group testing methods, and algorithms based on belief propagation. Three typical algorithms based on greedy pursuit are the Orthogonal Matching Pursuit (OMP), the Regularized OMP (ROMP) and the Stagewise OMP (StOMP) [1, 3 7]. The computational complexity of OMP strategies depends on the number of iterations needed for exact reconstruction, which is significantly smaller than that of linear program based methods. However, the stability of the pursuit algorithms is poor. As a possible remedy, the subspace pursuit algorithm is introduced for reconstruction of radar image in this paper. This method exhibits low reconstruction complexity, but has good reconstruction capability compared to that of linear programming method.

The input data for compressed sensing based reconstruction of radar image is the scattering field of the geometries, which could be computed by many numerical algorithms in computational electromagnetics [8]. In order to efficient compute the far field scattering over broad frequency- and angular- band, the physical optics (PO) [9] method is applied in this paper.

The remainder of this paper is organized as follows. Section II demonstrates the basic theory of compressive sensing technique applied in radar imaging. The theory of subspace pursuit method is discussed in section III. Numerical results of two geometries are presented to demonstrate the efficiency of this proposed method in Section IV. Conclusion is provided in Section V.

\section{BASIC THEORY OF COMPRESSIVE RADAR IMAGING}

The geometry of the radar imaging of a target is shown in Fig .1. The target is described in Cartesian coordinates, with its origin located at geometric center. The rotation rate of the target with respect to the radar is $\omega$. The range between the radar and the target center is $r_{0}$. Assume the range between the point $P(x, y)$ and the center is $r$, at an arbitrary time instant $t$, the range between one point $P(x, y)$ of the target and the radar is

$$
R=\sqrt{r^{2}+r_{0}^{2}+2 r r_{0} \sin (\theta+\omega t)}
$$

Then we model the signal received by radar receiver through the range $R$, which is given as

$$
S_{r}\left(\lambda_{i}\right)=\mathrm{e}^{-\mathrm{j} \frac{4 \pi}{\lambda_{i}} \sqrt{r^{2}+r_{0}^{2}+2 r r_{0} \sin (\theta+\omega t)}}
$$

where $\lambda_{i}$ is defined as the wave number of incident wave. Obviously, this is the formulation in frequency domain. If radar transmit the LFM signal, the LFM echo can also be modeled as formulate (2) by Fourier transform. In this paper, the back-projection method is applied to obtain the high resolution image of geometries and physical optics method is used to efficiently compute the echo in frequency domain. 


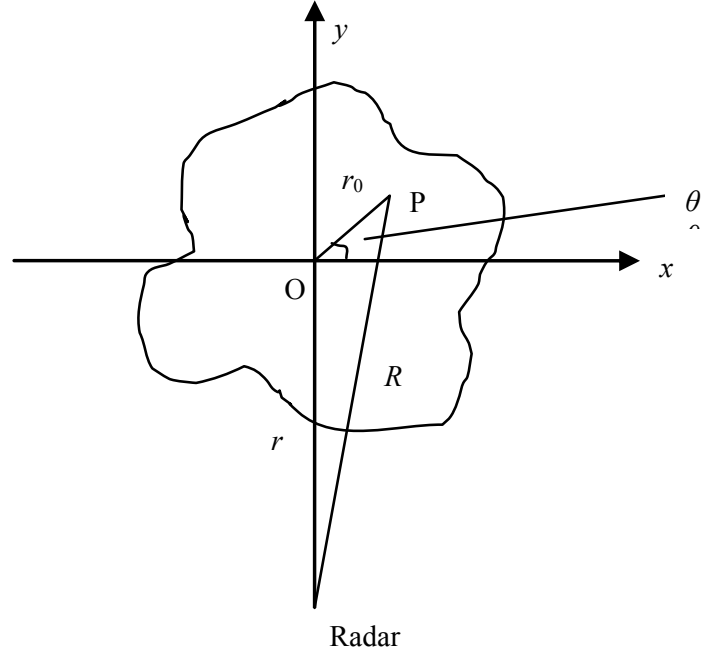

Figure 1. Radar imaging for rotation model

The sampling rate of a signal could be lower than Nyquist rate when using the compressive sensing technique. The basic point of it is the signal can be sparsely represented $[10,11]$. Accordingly, it is necessary to find firstly a suitable basis function to sparsely represent the signal. Take discrete-time signal $\mathbf{x}(n)$ with length of $N$ for example, $n=1,2,3 \ldots, N$.. Obviously, we can consider $\mathbf{x}$ as an $N \times 1$ column vector. The signal vector $\mathbf{x}$ is sparsely representable if there exists a sparsity basis $\boldsymbol{\Psi}^{T}=$ $\left\{\boldsymbol{\psi}_{1}, \quad \boldsymbol{\psi}_{2}, \ldots, \quad \boldsymbol{\psi}_{i}, \ldots, \boldsymbol{\psi}_{N}\right\}$ that provides a $K$-sparse representation of $\mathbf{x}$, that is

$$
\mathbf{x}=\sum_{i=1}^{N} \boldsymbol{\psi}_{i} \theta_{i}=\boldsymbol{\Psi} \boldsymbol{\theta}
$$

where $\mathbf{x}$ is a linear combination of $k$ basis vectors with nonzero coefficient. All the basis vectors are chosen from $\boldsymbol{\Psi}^{T}$ which is an $N \times N$ matrix. $\theta$ is the weighting coefficient as an $N \times 1$ column vector with $k$ nonzero elements. Rational choice of this sparsity basis to make $k$ as small as possible, it will not only help to improve the speed of signal receiver, but help to reduce the storage and transmission of signals in the system resource

\section{RECONSTRUCTION VIA SUBSPACE PURSUIT METHOD}

In our framework, the input data is the scattering field over a wide frequency- and angular-band which computed by physical optics. Obviously, the low-sampled input data corresponds to the observation vector in CS framework. The geometry is depicted as the combination of a number of spatial basis functions. The pulse function is used as the spatial basis functions. Since LFM signal is widely used for radar imaging, the testing function in this paper is not the Gaussian function but the LFM function with two important variables, the angle and the frequency, which means the radar transmits a wide band LFM signal to observe the geometry at each angle. In order to obtain the LFM echo, the Fourier transform is used to transfer scattering field from frequency domain to time domain. However, the sampling rate for both frequency- and angular- domain is much lower than Nyquist rate. Accordingly, a reconstruction algorithm based on CS is necessary to achieve this target.
The OMP-type methods [3] are suitable to be applied to solve the reconstruction problem. In this paper, the subspace pursuit method [12] is chosen since its reliability and efficiency. The basic theory of the subspace pursuit method for reconstruction of radar image comes from coding theory. Different from traditional OMP method, one starts by selecting the set of $\mathrm{k}$ most reliable information symbols. In our problem, the first $\mathrm{k}$ basis functions are selected according to the information from the observation vector. Then, some of the low-reliability functions from the $\mathrm{k}$ basis functions are retired and some novel informative functions chosen from other basis functions are selected. The coefficients of these basis are computed by solving linear equations. After several iterations, the final results depict the image of the geometry. Following is the detailed algorithm of the SP method.

\section{Subspace pursuit algorithm}

Input: $k, \boldsymbol{\Phi}, \mathbf{y}$

Initialization:

(1) $\mathbf{T}^{0}=\{k$ indices corresponding to the largest magnitude entries in the vector $\left.\boldsymbol{\Phi}^{*} \mathbf{y}\right\}$.

(2) $\mathbf{y}_{\mathrm{r}}^{0}=\operatorname{residual}\left(\mathbf{y}, \boldsymbol{\Phi}_{T^{0}}\right)$.

Iteration: At the $l^{\text {th }}$ iteration, go through the following steps

(1) $\mathbf{T}^{l}=\mathbf{T}^{l-1} \cup\{k$ indices corresponding to the largest magnitude entries in the vector $\left.\boldsymbol{\Phi} \cdot \mathbf{y}_{r}^{l-1}\right\}$.

(2) Set $\mathbf{x}_{p}=\boldsymbol{\Phi}_{T^{l}} \mathbf{y}$.

(3) $\mathbf{T}^{l}=\{K$ indices corresponding to the large elements of $\left.\mathbf{x}_{p}\right\}$.

(4) $\mathbf{y}_{r}^{l}=\operatorname{residual}\left(\mathbf{y}, \boldsymbol{\Phi}_{\mathbf{T}^{l}}\right)$.

(5) If $\left\|\mathbf{y}_{r}^{l}\right\|_{2}>\left\|\mathbf{y}_{r}^{l-1}\right\|_{2}$, let $\mathbf{T}^{l}=\mathbf{T}^{l-1}$ and quit the iteration

Output:

(1) The estimated signal $\mathbf{x}$, satisfying $\mathbf{x}_{\{1, \ldots, N\}-\mathbf{T}^{l}}=0$ and $\mathbf{x}_{\mathbf{T}^{l}}=\boldsymbol{\Phi}_{\mathbf{T}^{l}} \mathbf{y}$.

The range profile can be reconstructed by SP method. In [12], the reconstruction complexity of the SP algorithm is $O(m N \log K)$. There is a slightly higher for the complexity of SP for LFM signals in our work. Then, the traditional back-projection (BP) method is used to recover the 2-D image.

\section{NUMERICAL RESULTS}

In this section, a number of numerical results are presented to demonstrate the accuracy and efficiency of the proposed method for radar imaging of 3-D geometries. The subspace pursuit algorithm is applied to solve linear systems constructed by compressive sensing technique. All experiments are conducted on an Intel Core(TM) II Duo with $3.45 \mathrm{~GB}$ local memory and run at $2.40 \mathrm{GHz}$ in single precision. The iteration process of the solver is terminated when the 2-norm residual error is reduced by $10^{-3}$.

First of all, First of all, the radar images of two geometries, PEC Ogive and Double-Ogive [13], are analyzed in different frequency band. The detailed information about the structure of the two geometries can be referenced in [13]. The input data is the scattering of the geometries excited by plane wave. The scattering field is 
computed by physical optics method since the incident frequency band is C-band and K-band. In this two frequency bands, the objects can be considered as electrically large object which the assumption of high frequency is available. Using the physical optics, the current on the PEC surface of the object is computed by

$$
\mathbf{J}=2 \hat{\mathbf{n}} \times \mathbf{H}^{i}
$$

According to high frequency assumptions, the currents on the surfaces which are not illuminated by incident wave are considered to be zero. In order to judge the illuminated regions when using the $\mathrm{PO}$ approach to analyze the scattering of complicated objects, a special process is required at the very beginning.

From Fig .2-5, it is obvious that compressive sensing can lead to almost the same results as traditional BP method. In the C-band, the bandwidth is $4 \mathrm{GHz}$. According to Nyquist rate, the number of sampling points for traditional method is 41 . Obviously, the object is sparse in the region and only 21 samples are needed for compressive sensing method. This sampling rate is much smaller than Nyquist rate. The same conclusions can be made in the Kband. The bandwidth is $9 \mathrm{GHz}$ and the number of samples is 91 under Nyquist rate. When compressive sensing method is used, only 31 samples is required and the subspace pursuit method is able to recover the geometries precisely.

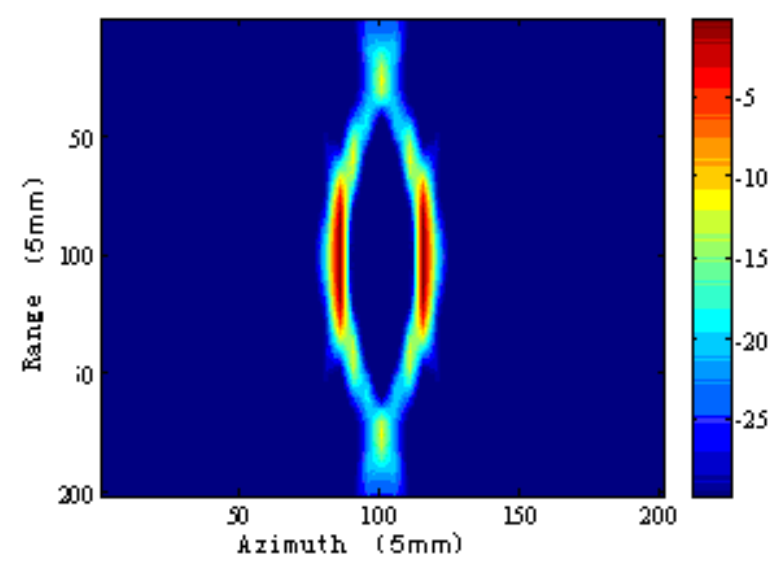

(a)

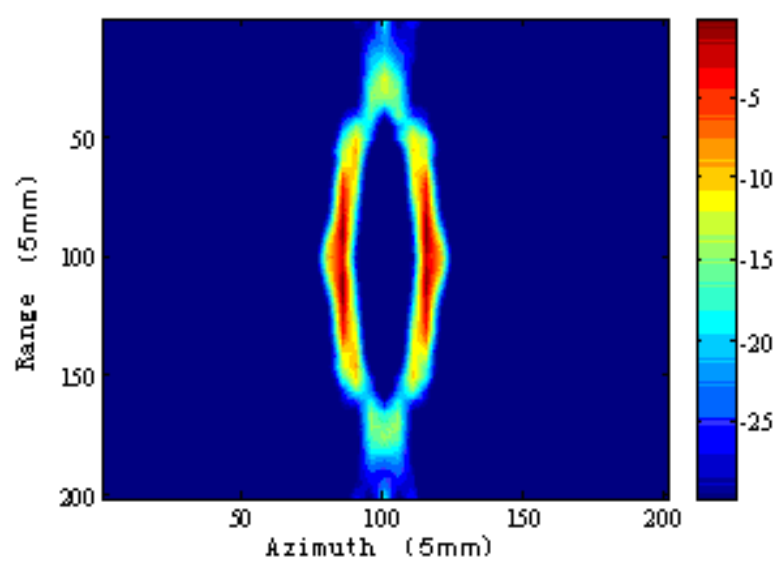

(b)

Figure 2. Radar image of Ogive with $4 \mathrm{GHz}$ bandwidth at C-band: (a) traditional BP method; (b) compressive sensing via subspace pursuit

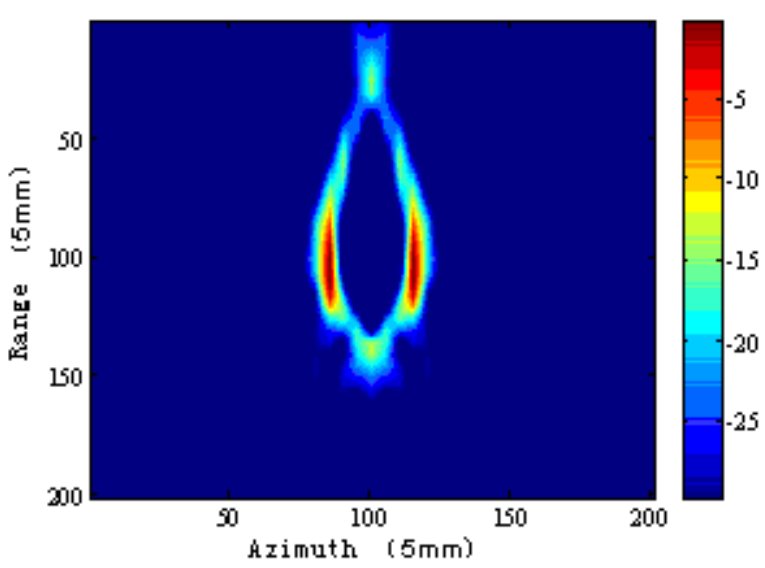

(a)

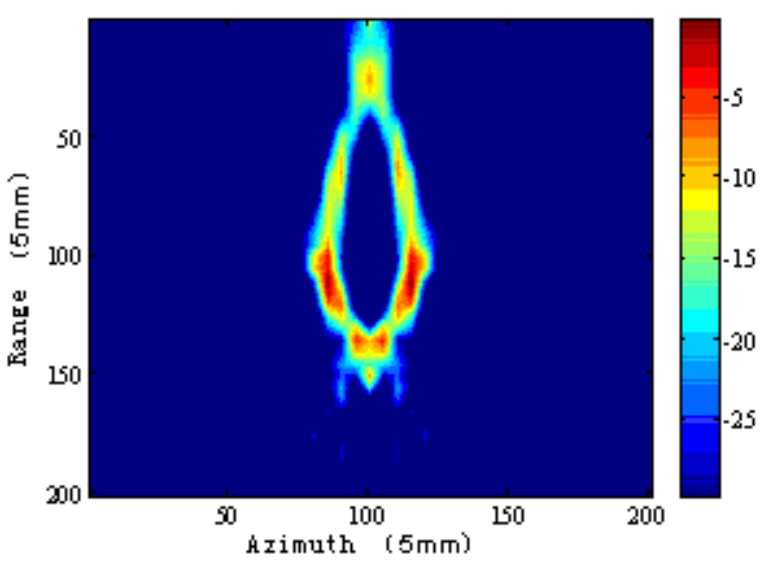

(b)

Figure 3. Radar image of Double-Ogive with $4 \mathrm{GHz}$ bandwidth at $\mathrm{C}$ band: (a) traditional BP method; (b) compressive sensing via subspace pursuit

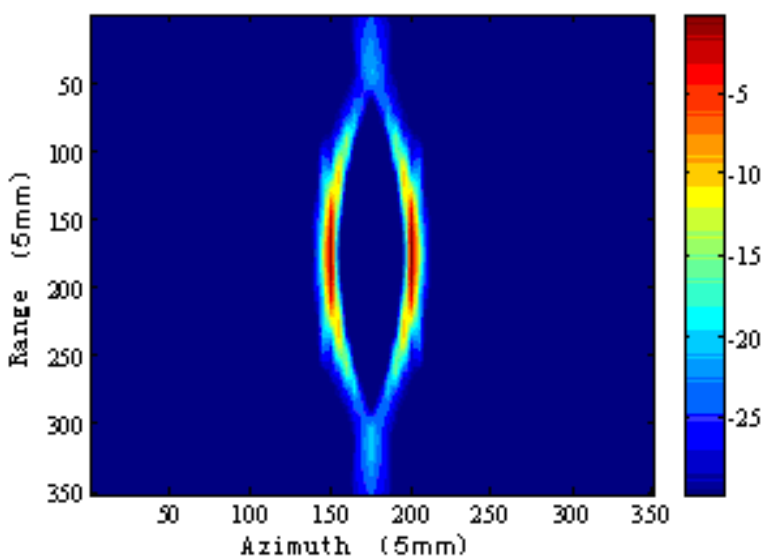

(a) 


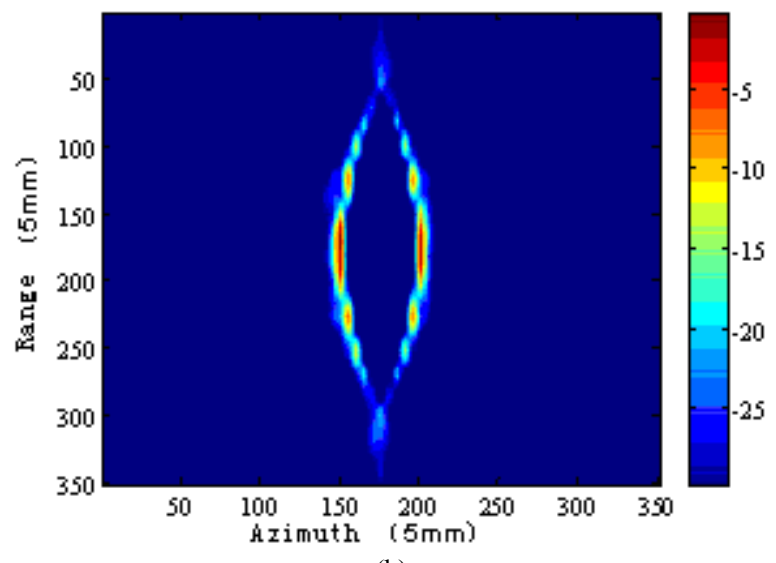

(b)

Figure 4. Radar image of Ogive with $9 \mathrm{GHz}$ bandwidth at K-band: (a) traditional BP method; (b) compressive sensing via subspace pursuit

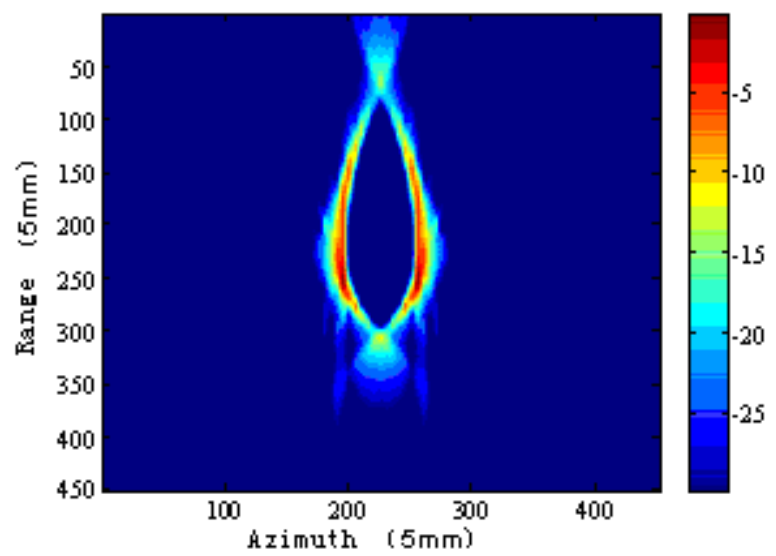

(a)

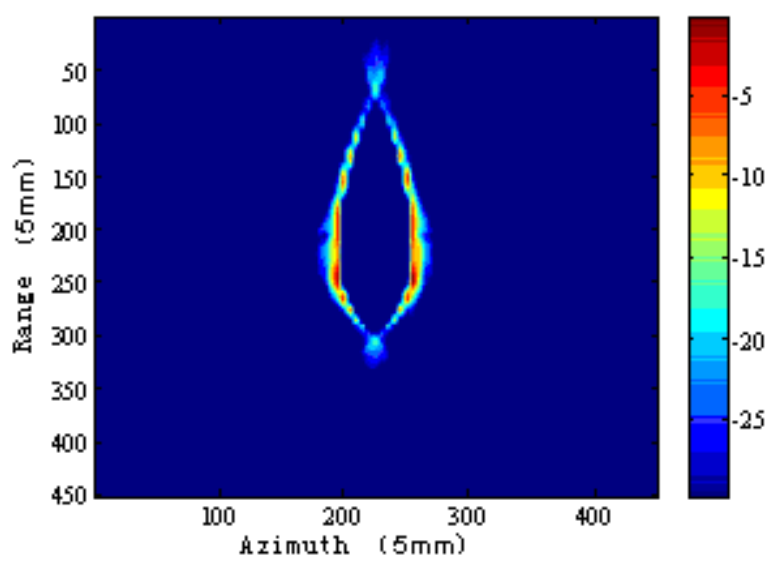

(b)

Figure 5. Radar image of Double-Ogive with $9 \mathrm{GHz}$ bandwidth at Kband: (a) traditional BP method; (b) compressive sensing via subspace pursuit

\section{CONCLUSIONS}

Compressed sensing is a new technique for signal processing. It can achieve high-resolution reconstruction with low Nyquist sampling rate and non-adaptive sampling for the signal. Compressed sensing technique could be used in radar systems, which can significantly reduce the power consumption of the transmitter and receiver, volume and weight, so as to achieve lower costs. This paper has focused on the applications of compressed sensing technique in radar systems, which emphasis on the compressed radar imaging algorithm for sparse scene. By using the subspace pursuit method, the radar image can be recovered efficient and robust. A large number of simulation examples, whose scattering field are computed by the physical optics, can validate the compressive radar imaging algorithm based on subspace pursuit method. Compared with the traditional imaging method, using compressed sensing technique can not only sample the SAR echo data below the Nyquist sampling rata, but also can effectively reduce the generation of sidelobe.

\section{ACKNOWLEDGMENT}

One of us thanks the support of National Natural Science Foundation of China (No: 61261005), Open Project of National Key Laboratory (No: K201326)

\section{REFERENCES}

[1] D. L. Donoho, "Compressed sensing," IEEE Trans. Information Theory, vol.52, no.4, pp.1289 1306, April 2006

[2] Emmanuel J. Candes, Justin Romberg, Terence Tao, "Robust uncertainty principles: Exact signal reconstruction from highly incomplete frequency information," IEEE Trans. Information Theory, vol.52, no.2, pp.489 509, February 2006

[3] Joel A. Tropp, Anna C. Gilert, "Signal recovery from random measurements via orghogonal matching pursuit," IEEE Trans Information Theory, vol.53, no.12, pp.4655 4666, December 2007

[4] W. Dai and O. Milenkovic, "Subspace pursuit for compressive sensing: Closing the gap between performance and complexity,' submitted for publication, March 2008

[5] M. Rudelson and R. Bershynin, "Sparse reconstruction by convex relaxation: Fourier and Gaussian measurements," In Proc. 40th Annual Conference on Information Sciences and Systems, Princeton, March 2006

[6] M. A. T Figueiredo, R. D. Nowak, S. J. Wright, "Gradient projection for sparse reconstruction: Application to compressed sensing and other inverse problems," IEEE J. Selected Topics in Signal Processing: Special Issue on Convex Optimization Methods for Signal Processing, vol. 1, no. 4, pp. 586 598, 2007

[7] A. Gilbert, M. Strauss, J. Tropp, R. Vershynin, "One skethc for all: Fast algorithm for compressed sensing," In Proc. 39th ACM Symp. Theory of Computing, San Diego, June 2007

[8] R. F. Harrington. Field computation by moment methods. Malabar, Fla.: R. E. Krieger, 1968

[9] N. N. Youssef, "Radar cross section of complex targets," Proc. IEEE, vol.77, no.5, pp.722 734, May 1989.

[10] Richard G. Baraniuk, "Compressive sensing," IEEE Signal Processing Magazine, pp.118 124, July 2007

[11] Justin Romberg, "Imaging via compressive sampling Introduction to compressive sampling and recovery via convex programming," IEEE Signal Processing Magazine, pp.14 20, March 2008

[12] Wei Dai, Olgica Milenkovic, "Subspace pursuit for compressive sensing signal reconstruction," IEEE Trans. Information Theory, vol. 55, no. 5, pp. $2230 \sim 2249$, May 2009 .

[13] Alex C. Woo, Helen T. G. Wang, Michael J. Schuh, Michael L. Sanders, "Benchmark radar targets for the validation of computational electromagnetics programs," IEEE Antennas and Propagation Magazine, vol. 35, no. 1, pp. $84 \sim 89$, February 1993. 